\title{
Base editors: a powerful tool for generating animal models of human diseases
}

\author{
Zongyang Lu ${ }^{1,2}$ and Xingxu Huang ${ }^{1,3, *}$ \\ ${ }^{1}$ School of Life Science and Technology, ShanghaiTech University, 100 Haike Rd., Pudong New Area, Shanghai 201210, China. \\ ${ }^{2}$ University of Chinese Academy of Sciences, 100049 Beijing, China. \\ ${ }^{3}$ CAS Center for Excellence in Molecular Cell Science, Shanghai Institute of Biochemistry and Cell Biology, Chinese Academy of \\ Sciences; University of Chinese Academy of Sciences, 320 Yueyang Road, Shanghai 200031, China. \\ * Corresponding Authors: \\ Xingxu Huang Ph.D, Principal Investigator, Professor; School of Life Science and Technology, ShanghaiTech University, Room 411B, \\ No. 11 Building, 100 Haike Rd., Pudong New Area, Shanghai 201210, China; E-mail: huangxx@shanghaitech.edu.cn
}

\begin{abstract}
Myriads of genetic mutations, including base substitutions, deletions, and insertions as well as chromosome structural variations, have been detected in many human diseases. Although current combination of genomics and bioinformatics has contributed greatly to understanding the genetics of these disorders, it remains challenging to ensure the causal functions of each mutation, and then to further investigate the underlying mechanism and to develop therapeutic strategies. Animal models generated by genome engineering are the key to address these issues. In this review, we will first revisit the limitation of conventional gene editing tools and mouse models generated in the past. We will then introduce a novel tool, base editors (BEs), which present a new promising approach to establish pathogenically relevant animal models. Finally, we will discuss the application of BEs in non-human primates and share our perspectives on future development of base-editing techniques.
\end{abstract}

\section{BASE EDITORS: A NOVEL GENOME ENGINEERING TOOL}

Genetically engineered mouse models of human diseases have long been used in understanding the underlying mechanisms of the clinically relevant genetic disorders. In the past, using gene-targeting technology to establish animal models is time-consuming and laborious. Recently developed genome editing tools, including ZFNs (zincfinger nucleases), TALENs (transcription activator-like effector nucleases) and CRISPR (clustered regularly interspaced short palindromic repeat), facilitate the generation of mouse models. However, although very useful, ZFNs and TALENs still suffered from two limitations. First, ZF (zincfinger) protein and TALE (transcription activator-like effec- tor) protein need to be fused to a nuclease, such as fok1. Fok1 function as a dimmer, therefore, pairs of ZF or TALE proteins are essential but sometimes tough to generate. Second, ZFNs- and TALENs-based genome targeting requires ZFN or TALE assembly for each targeted sequence, which is much less convenient than CRISPR, which needs only a short sgRNA (single-guided RNA). For these reasons, CRISPR has been broadly used for gene function annotation. Nevertheless, CRISPR/Cas9-assisted homologous recombination works poorly in modeling clinically relevant point mutations. Methods to precisely and efficiently introduce base substitutions across the genome has been long awaited.

Novel single base editing tools named BEs (base editors) created by fusing the cytosine/adenine deaminase to the $\mathrm{N}$-terminus of Cas 9 nickase have been constructed to convert $A$ to $G$ or $C$ to $T$ at specific sites, without causing DSB (double-strand breaks) and with minimized off-target [1] (Fig. 1). Based on the fused deaminase enzyme, BEs can be categorized into $C B E$ (cytosine base editor) and $A B E$ (adenine base editor). For CBE, the rat APOBEC1 (rAPOBEC1) deaminase and sea lamprey AID ortholog (PmCDA1) deaminase are fused to Cas9 nickase, respectively [2, 3]. CBEs have been used in various species [4-6], including human embryos $[7,8]$. To perform $A$ to $G$ conversion, $A B E$ was engineered by fusing one natural adenine deaminase (ecTadA) and one adenine deaminase variant (ecTadA*) by protein evolution to the $\mathrm{N}$-terminus of Cas9 nickase (Fig. 1). Both $B E 3$ and $A B E$ have an efficient editing window of approximately five $n t$, typically from $C_{4} / A_{4}$ to $C_{8} / A_{8}$ distal to PAM (Fig. 1).

Comment on Liu Z, Lu Z, Yang G, Huang S, Li G, Feng S, Liu Y, Li J, Yu W, Zhang Y, Chen J, Sun Q, Huang X (2018). Efficient generation of mouse models of human diseases via ABE- and BE-mediated base editing. Nat Commun 9(1):2338. doi: 10.1038/s41467-018-04768-7 


\section{BE3}

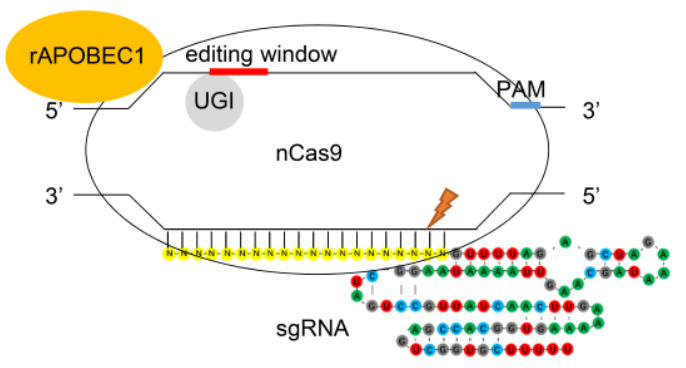

ABE



FIGURE 1: Targeted base substitutions without double strand break by cytidine- and adenine- deaminase. In these illustrations of base editing, deaminase proteins are shown in orange. The base-editing windows are highlighted in red, and the PAM sequences in blue on the DNA strand. nCas9 indicates Cas9 nickase harboring D10A mutation. Left: BE3 composed of rAPOBEC1, nCas9 and UGI (uracil glycosylase inhibitor). rAPOBEC1 indicates cytidine deaminase APOBEC1 derived from rat. Right: ABE composed of ecTadA (WT)-ecTadA* (7.10) fusion and nCas9. ecTadA (WT) indicates natural adenine deaminase Escherichia coli TadA. ecTadA* (7.10) indicates Escherichia coli TadA variant after protein evolution.

BEs have accelerated the generation of clinically more relevant mouse models of human diseases caused by single-nucleotide substitutions [9]. Base substitutions (between $C / G$ and $T / A$ ) account for $~ 54 \%$ known pathogenic human SNPs, suggesting the huge potential of BEs in modeling and correcting disease-associated mutations [1]. In our studies, we chose androgen receptor $(A r)$ and homeobox D13 (Hoxd13) as targeted genes, and generated two mouse models, androgen insensitivity syndrome (AIS) and Syndactyly, by introducing $A$ to $G$ conversions at corresponding homologous pathogenic sites of patients through microinjection of mRNA and sgRNAs into zygotes. The Syndactyly model harboring modifications on Hoxd13 was developed with germline transmission, demonstrating introduced mutations are heritable. Furthermore, considering different types of substitutions (including $A$ to $G$ and $C$ to $T$ ) usually co-exist in same patients in clinic, we introduced $A$ to $G$ and $C$ to $T$ substitutions simultaneously in vivo. To avoid possible competition between BEs, we applied CBE-SaCas9 together with ABE-SpCas9, and successfully generated two types of substitutions at different targeted loci (Hoxd13 and Tyr) simultaneously. As expected, the founder pups harbored two kinds of phenotypes, fused digits (Hoxd13) and white coat (Tyr), indicating the success in modeling complex diseases from multiple mutations. Furthermore, comprehensive off-target analysis by WGS and targeted deep sequencing analysis revealed no detectable off-target effects. Meanwhile, several groups also utilized BEs to generate premature stop codon to precisely disrupt endogenous genes [6, 10], further demonstrating the versatility of BEs in modeling disease-relevant mutations. These studies pave the way to explore more than 30000 pathogenic SNPs across the genome

\section{NON-HUMAN PRIMATE MODELS TO RECAPITULATE HUMAN DISEASES}

As we known, non-human primates (NHPs) are considered as one of the best disease model organisms due to their similarities to humans in genetics, anatomy and physiology $[11,12]$. Recent advances have proved the great promise of NHP models harboring genome modifications in biomedical research [13-15]. We believe base editing in nonhuman primates will better mimic human diseases. By genome sequencing and comparison, NHPs displayed high similarity with humans on drug targets and homologous disease genes [16]. We also analyzed the whole-genome sequences of human and crab-eating macaque from NCBI database, and found the number of codons (excluding start and stop codons) across whole genome of humans and crab-eating macaque are quite similar. There are about $1.2 \times 10^{7}$ in humans and $1.1 \times 10^{7}$ in crab-eating macaque, and most of them (1.0 $\times 10^{7}$ codons) homologous (Fig. 2A). In addition, the editable codons for $\mathrm{BE} 3$ and $\mathrm{ABE}$ are more than $1.49 \times 10^{6}$ in human and macaque, and most of them $\left(\sim 1.40 \times 10^{6}\right)$ are homologous too, indicating the high feasibility for modelling pathogenic mutations in NHPs using BEs (Fig. 2A).

\section{PROBLEMS AND PERSPECTIVES}

Although BEs show their versatility in genome manipulation, two main concerns remain to be addressed. First, BEs are limited by PAM (protospacer-adjacent motif) and editing windows, therefore cannot cover the majority of sites of interest including many pathogenic sites. Increasing the genome-targeting scope of BEs is urgent. To address this concern, cytidine deaminase fusions with SaCas9 (PAM: NNGRRT) and Cpf1 (PAM: TTTN), respectively, have recently been developed $[17,18]$. We previously developed a BEPLUS tool with broadened editing window at targeted sites 
A
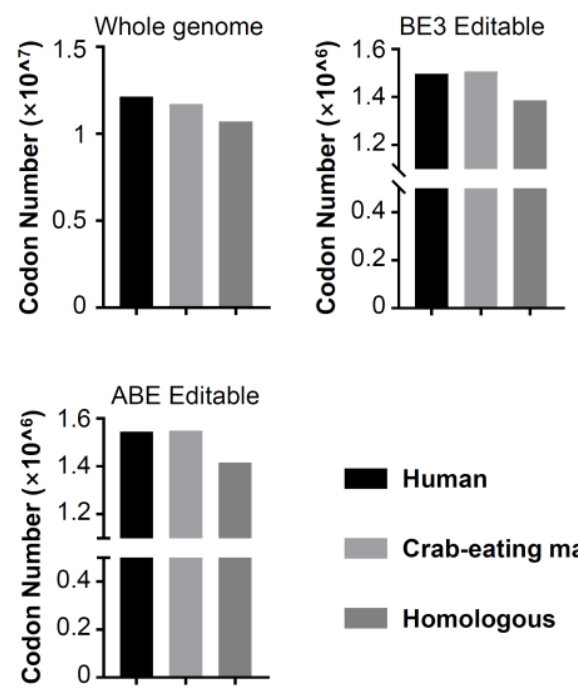

B

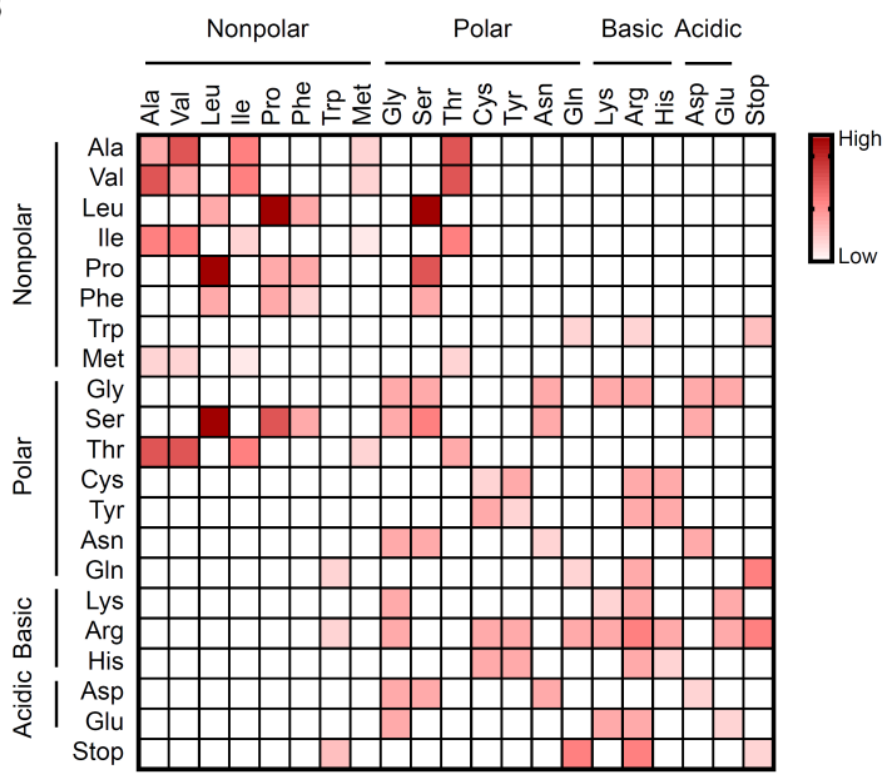

FIGURE 2: The analysis of editable codons for BEs. (A) The number of codons from NCBI database. Histograms in this panel show the number of codons of human and crab-eating macaque genomes as well as their homologous codons (Whole genome), and the number of codons from these two species editable with $B E 3$ and $A B E$ (BE3 Editable and $A B E$ Editable, respectively). (B) Illustration of the repertoire of the amino acid conversions generated by BEs (BE3 and $A B E)$. The amino acids on $x$ axis and $y$ axis are converted between each other. The theoretical abilities of amino acid conversions are displayed in red-white color scale.

by using Suntag tail for recruiting 10 copies of APOBECUGI-GB1 [19]. A recently evolved Cas9 variant, xCas9, even provides more choices because of its broader PAM compatibility [20]. Second, both A-to-G and C-to-T are base substitutions within pyrimidines or purines. However, base transversions (changes between pyrimidines and purines) account for a larger proportion of diseases. To date, it is hard to achieve base tranversions, because pyrimidines and purines have totally different molecular structures. As shown in Fig. 2B, the available amino acid substitutions generated by current BEs only account for $19 \%$ (80/420) of the missense mutations for the limitation of BEs that the mutated codons are only derived from pyrimidines/purines-to-pyrimidines/purines conversions. An efficient base transversion method will be highly desirable.

In conclusion, BEs have been successfully used to precisely edit the genomes of several organisms, including mammals, and have proven to be a powerful tool for modeling human diseases caused by single-base mutations. The applications of BEs in NHPs will provide us better models of human diseases and shed new light on our understanding of their pathophysiology.

\section{REFERENCES}

1. Gaudelli NM, Komor AC, Rees HA, Packer MS, Badran AH, Bryson DI, Liu DR (2017). Programmable base editing of $A^{*} T$ to $G^{*} C$ in genomic

\section{ACKNOWLEDGEMENTS}

We thank members of the Huang laboratory for helpful discussions. We are grateful to Dr. Hongjun Liu from ShanghaiTech University for excellent manuscript editing. This works is supported by National Science Foundation of China (81671516).

\section{CONFLICT OF INTEREST}

The authors declare no conflict of interest.

\section{COPYRIGHT}

(C) 2018 Lu and Huang. This is an open-access article released under the terms of the Creative Commons Attribution (CC BY) license, which allows the unrestricted use, distribution, and reproduction in any medium, provided the original author and source are acknowledged.

Please cite this article as: Zongyang Lu and Xingxu Huang (2018). Base editors: a powerful tool for generating animal models of human diseases. Cell Stress 2(10): 242-245. doi: 10.15698/cst2018.10.156

DNA without DNA cleavage. Nature 551(7681): 464-471. doi $10.1038 /$ nature24644 
2. Komor AC, Kim YB, Packer MS, Zuris JA, Liu DR (2016). Programmable editing of a target base in genomic DNA without double-stranded DNA cleavage. Nature 533(7603): 420-424. doi: 10.1038/nature17946

3. Nishida K, Arazoe T, Yachie N, Banno S, Kakimoto M, Tabata M, Mochizuki M, Miyabe A, Araki M, Hara KY, Shimatani Z, Kondo A (2016). Targeted nucleotide editing using hybrid prokaryotic and vertebrate adaptive immune systems. Science 353(6305). doi: $10.1126 /$ science.aaf8729

4. Shimatani Z, Kashojiya S, Takayama M, Terada R, Arazoe T, Ishii H, Teramura H, Yamamoto T, Komatsu H, Miura K, Ezura H, Nishida K, Ariizumi T, Kondo A (2017). Targeted base editing in rice and tomato using a CRISPR-Cas9 cytidine deaminase fusion. Nat Biotechnol 35(5):441-443. doi: 10.1038/nbt.3833

5. Zong Y, Wang Y, Li C, Zhang R, Chen K, Ran Y, Qiu JL, Wang D, Gao C (2017). Precise base editing in rice, wheat and maize with a Cas9cytidine deaminase fusion. Nat Biotechnol 35(5):438-440. doi: $10.1038 / \mathrm{nbt} .3811$

6. Kim K, Ryu SM, Kim ST, Baek G, Kim D, Lim K, Chung E, Kim S, Kim JS (2017). Highly efficient RNA-guided base editing in mouse embryos. Nat Biotechnol 35(5):435-437. doi: 10.1038/nbt.3816

7. Li G, Liu Y, Zeng Y, Li J, Wang L, Yang G, Chen D, Shang X, Chen J, Huang $X$, Liu J (2017). Highly efficient and precise base editing in discarded human tripronuclear embryos. Protein Cell 8(10):776-779. doi: 10.1007/s13238-017-0458-7

8. Liang $P$, Ding $C$, Sun $H$, Xie $X, X u Y$, Zhang $X$, Sun $Y$, Xiong $Y$, Ma W, Liu $Y$, Wang $Y$, Fang J, Liu D, Songyang Z, Zhou C, Huang J (2017). Correction of beta-thalassemia mutant by base editor in human embryos. Protein Cell 8(11):811-822. doi: 10.1007/s13238-017-0475-6

9. Liu Z, Lu Z, Yang G, Huang S, Li G, Feng S, Liu Y, Li J, Yu W, Zhang Y, Chen J, Sun $Q$, Huang $X$ (2018). Efficient generation of mouse models of human diseases via ABE- and BE-mediated base editing. Nature Communications 9(1): 2338. doi: 10.1038/s41467-018-04768-7

10. Kuscu C, Parlak M, Tufan T, Yang J, Szlachta K, Wei X, Mammadov R, Adli M (2017). CRISPR-STOP: gene silencing through base-editinginduced nonsense mutations. Nat Methods. doi: 10.1038/nmeth.4327

11. Chan AW (2004). Transgenic nonhuman primates for neurodegenerative diseases. Reprod Biol Endocrinol 2:39. doi: 10.1186/14777827-2-39

12. Izpisua Belmonte JC, Callaway EM, Caddick SJ, Churchland P, Feng G, Homanics GE, Lee KF, Leopold DA, Miller CT, Mitchell JF, Mitalipov
S, Moutri AR, Movshon JA, Okano $H$, Reynolds JH, Ringach $D$, Sejnowski TJ, Silva AC, Strick PL, Wu J, Zhang F (2015). Brains, genes, and primates. Neuron 86(3): 617-631. doi: 10.1016/j.neuron.2015.03.021

13. Wan H, Feng C, Teng F, Yang S, Hu B, Niu Y, Xiang AP, Fang W, Ji W, Li W, Zhao X, Zhou Q (2015). One-step generation of $p 53$ gene biallelic mutant Cynomolgus monkey via the CRISPR/Cas system. Cell Res 25(2): 258-261. doi: 10.1038/cr.2014.158

14. Zhao $H$, Tu Z, Xu H, Yan S, Yan H, Zheng Y, Yang W, Zheng J, Li Z, Tian R, Lu Y, Guo X, Jiang YH, Li XJ, Zhang YQ (2017). Altered neurogenesis and disrupted expression of synaptic proteins in prefrontal cortex of SHANK3-deficient non-human primate. Cell Res 27(10): 1293-1297. doi: 10.1038/cr.2017.95

15. Niu Y, Shen B, Cui Y, Chen Y, Wang J, Wang L, Kang Y, Zhao X, Si W, Li W, Xiang AP, Zhou J, Guo X, Bi Y, Si C, Hu B, Dong G, Wang H, Zhou Z, Li T, Tan T, Pu X, Wang F, Ji S, Zhou Q, Huang X, Ji W, Sha J (2014). Generation of gene-modified cynomolgus monkey via Cas9/RNAmediated gene targeting in one-cell embryos. Cell 156(4): 836-843. doi: 10.1016/j.cell.2014.01.027

16. Yan G, Zhang G, Fang X, Zhang Y, Li C, Ling F, Cooper DN, Li Q, Li Y, van Gool AJ, Du H, Chen J, Chen R, Zhang P, Huang Z, Thompson JR, Meng $Y$, Bai $Y$, Wang J, Zhuo $M$, Wang $T$, Huang $Y$, Wei L, Li J, Wang $Z$, Hu H, Yang P, Le L, Stenson PD, Li B, et al. (2011). Genome sequencing and comparison of two nonhuman primate animal models, the cynomolgus and Chinese rhesus macaques. Nat Biotechnol 29(11): 10191023. doi: 10.1038/nbt.1992

17. Kim YB, Komor AC, Levy JM, Packer MS, Zhao KT, Liu DR (2017). Increasing the genome-targeting scope and precision of base editing with engineered Cas9-cytidine deaminase fusions. Nat Biotechnol 35(4): 371-376. doi: 10.1038/nbt.3803

18. Li X, Wang Y, Liu Y, Yang B, Wang X, Wei J, Lu Z, Zhang Y, Wu J, Huang $X$, Yang L, Chen J (2018). Base editing with a Cpf1-cytidine deaminase fusion. Nat Biotechnol 36(4): 324-327. doi: 10.1038/nbt.4102

19. Jiang W, Feng S, Huang S, Yu W, Li G, Yang G, Liu Y, Zhang Y, Zhang L, Hou Y, Chen J, Chen J, Huang X (2018). BE-PLUS: a new base editing tool with broadened editing window and enhanced fidelity. Cell Res. doi: 10.1038/s41422-018-0052-4

20. Hu JH, Miller SM, Geurts MH, Tang W, Chen L, Sun N, Zeina CM, Gao X, Rees HA, Lin Z, Liu DR (2018). Evolved Cas9 variants with broad PAM compatibility and high DNA specificity. Nature 556(7699): 57-63. doi: $10.1038 /$ nature26155 